\title{
FINITE SIMPLE GROUPS CONTAINING A SELF-CENTRALIZING ELEMENT OF ORDER 6
}

\author{
JOHN L. HAYDEN AND DAVID L. WINTER
}

\begin{abstract}
By a self-centralizing element of a group we mean an element which commutes only with its powers. In this paper we establish the following result:

THEOREM. Let $G$ be a finite simple group which has a self-centralizing element of order 6 . Assume that $G$ has only one class of involutions. Then $G$ is isomorphic to one of the groups $M_{11}, J_{1}, L_{3}(3), L_{2}(11), L_{2}(13)$.
\end{abstract}

By a self-centralizing (s.c.) element of a group we mean an element which commutes only with its own powers. The structure of a finite group containing a s.c. element of order 2 has been known for a long time. The classification of finite groups with a s.c. element of order 3 was carried out by Feit and Thompson [2] and is an important tool in this paper. The determination of finite simple groups containing a s.c. element of prime order $p>3$ is a classical problem as yet unsolved although some special cases have been handled. In [12], M. Suzuki proved that the only finite simple groups containing a s.c. element of order 4 are $L_{2}(7), A_{6}$ and $A_{7}$. Finite simple groups containing a s.c. element of order 8 are studied in [10]. In this paper we establish the following result.

THEOREM. Let $G$ be a finite simple group which has a self-centralizing element of order 6. Assume that $G$ has only one class of involutions. Then $G$ is isomorphic to one of the groups $M_{11}, J_{1}, L_{3}(3), L_{2}(11), L_{2}(13)$.

The alternating groups $A_{8}, A_{9}$ are examples of simple groups with a s.c. element of order 6 which has two classes of involutions. As indicated in [8], it may be very difficult to handle groups containing a s.c. element of order 6 having more than one class of involutions.

The smallest Ree group is an example of a nonsimple group containing a s.c. element of order 6 and having only one class of involutions. It is isomorphic to an extension of $L_{2}\left(2^{3}\right)$ by a field automorphism of order 3 .

We shall make use of the following theorem.

(1.1) ([2]). If $Y$ is a finite group with a s.c. element of order 3, then $Y$ has a normal subgroup $N$ such that one of the following holds:

(i) $Y / N$ is cyclic of order 3 and $N$ is nilpotent.

(ii) $Y / N$ is dihedral of order 6 and $N$ is nilpotent.

Received by the editors December 6, 1976 and, in revised form, March 25, 1977.

AMS (MOS) subject classifications (1970). Primary 20D05. 
(iii) $Y / N \cong A_{5}$ and $N$ is an elementary abelian 2-group.

(iv) $Y / N \cong L_{2}(7)$ and $N=1$.

From now on we shall let $G$ denote a group satisfying the hypotheses of our theorem. Then $G$ contains an element $x$ of order 3 and an involution $t$ such that $[t, x]=1$ and $C(t x)=\langle t x\rangle$. Furthermore every involution of $G$ is conjugate to $t$.

(1.2) $C(x)=\langle t\rangle L$ where $L$ is a nilpotent normal subgroup of $C(x)$ of odd order. $L /\langle x\rangle$ is abelian and is inverted by $t$.

Proof. Let $\bar{C}=C(x) /\langle x\rangle$ and let $\bar{v} \in \bar{C}$ be such that $[\bar{v}, \bar{t}]=\overline{1}$. Then $\overline{[v, t]}=\overline{1}$ and so $[v, t]=x_{0} \in\langle x\rangle$. Hence $v^{-1} t v=x_{0} t$. Since $t$ has order 2, $x_{0}=1$ and $v \in C(t) \cap C(x)=\langle x t\rangle$. Hence $\bar{v} \in\langle\bar{t}\rangle$. Thus $\bar{t}$ is a s.c. element of $\bar{C}$ of order 2 . The structure of $\bar{C}$ is therefore known and (1.2) follows easily.

(1.3) Let $H$ be a subgroup of $G$ with $t x \in H$. Assume that $H$ contains $a$ normal 2-subgroup $U$ with $t \in U$. Then $C_{H}(x)=\langle t x\rangle$. Also if $\bar{H}=H / U$, then $\bar{x}$ is a s.c. element of $\bar{H}$ of order 3.

Proof. By (1.2), $U \cap C(x)=\langle t\rangle \triangleleft C_{H}(x)$. But by (1.2), $N_{C(x)}(\langle t\rangle)=\langle t x\rangle$. Hence $C_{H}(x)=\langle t x\rangle$. This implies that $\langle x\rangle$ is a Sylow 3-subgroup of $H$. It is well known and easily verified that $\overline{N(\langle x\rangle)}=N_{\bar{H}}(\langle\bar{x}\rangle)$. It is also easily checked from this that $\bar{y} \in \bar{H}$ centralizes $\bar{x}$ if and only if $y \in C_{H}(x) U$. Therefore $C_{\bar{H}}(\bar{x})=\overline{\langle t x\rangle U}=\langle\bar{x}\rangle$.

By setting $H=C(t), U=\langle t\rangle$, we have as an immediate consequence of (1.1) and (1.3)

(1.4) $C(t)$ contains a normal subgroup $N \geqslant\langle t\rangle$ such that one of the following holds:

(i) $C(t) / N$ is cyclic of order 3 and $N /\langle t\rangle$ is nilpotent.

(ii) $C(t) / N$ is dihedral of order 6 and $N$ is nilpotent.

(iii) $C(t) / N \cong A_{5}$ and $N /\langle t\rangle$ is an elementary abelian 2-group.

(iv) $C(t) / N \cong L_{2}(7)$ and $N=\langle t\rangle$.

The remainder of the proof of the theorem is divided into the four cases determined by (1.4). Assume first that (1.4) (iv) holds. Then $C(t) /\langle t\rangle \cong$ $L_{2}(7)$. It has been proved by Schur [11, p. 120], that $C(t)$ is either isomorphic to a direct product of $\langle t\rangle$ and $L_{2}(7)$ or to $S L(2,7)$. By a result of Janko and Thompson [9], the first case is not possible. In the second case $C(t)$, and hence $G$, has a quaternion Sylow 2-subgroup. By a well-known result of Brauer and Suzuki, $G$ cannot be simple. Therefore (1.4) (iv) cannot hold.

Assume next that (1.4) (iii) holds with $N=\langle t\rangle$. The argument of the preceding paragraph shows $C(t)=\langle t\rangle \times K, K \cong A_{5}$. Therefore, by [7] $G$ is isomorphic to $J_{1}$.

In the remaining cases $C(t)$ is solvable or $C(t) / N \cong A_{5}$. In the latter case $|N|>2$ with $C(N) \subseteq N$. Hence $C(t)$ is 2-constrained in all cases. As $G$ has 
only one class of involutions, a theorem of Gorenstein and Goldschmidt [3, p. 74] implies $O(C(t))=1$ or $S C N_{3}(2)=\varnothing$. In the latter case, Corollary 4, [5] shows $G$ is isomorphic to one of the groups $L_{2}(q), L_{3}(q), U_{3}(q), q$ odd, $U_{3}(4)$, $A_{7}$ or $M_{11}$. The only groups among these satisfying our hypotheses are $L_{3}(3)$, $M_{11}, L_{2}(11)$ and $L_{2}(13)$.

We may now assume $O(C(t))=1$. This forces $N$ to be a 2-group and $|C(t)|=2^{a} \cdot 3 \cdot 5^{b}$ for some $a, b$. We now claim that if $M$ is a 2-local subgroup of $G$, then each Sylow subgroup of $M$ of odd order is cyclic. Without loss we may assume $t \in Z\left(O_{2}(M)\right)=Z$. Suppose $M$ contains an elementary abelian $p$-subgroup $P$ of order $p^{2}, p$ odd. By a well-known result [4, p. 188], $Z=\Pi_{y \in P^{*}} C_{Z}(y)$. Again without loss, we can assume $t \in C_{Z}(y)$ for some $y \in P^{\#}$. Then $y \in C(t), p \in\{3,5\}$. Now let $y_{1} \in P-\langle y\rangle$. Since $Z \triangleleft M, t^{y_{1}} \in Z \cap C(t)$. Also, $\left(t^{y_{1}}\right)^{y}=\left(t^{y}\right)^{y_{1}}=t^{y_{1}}$ so $t^{y_{1}} \in C(t) \cap C(y)$. If $p=3,|y|=3$ and, as $\langle t y\rangle$ is conjugate to $\langle t x\rangle$ in $C(t), C(t) \cap C(y)=\langle t y\rangle$. If $p=5$, we are in case (1.4)(iii). Let $\bar{C}=C(t) /\langle t\rangle$. As $\bar{x}$ is a s.c. element of order 3 in $\bar{C}$, Theorem 8.2 of [6] implies $\bar{N}=N /\langle t\rangle$ is the direct sum of minimal normal subgroups of $\bar{C}$ of order 16 on which $A_{5} \cong S L(2,4)$ acts in the natural way. Consequently $\bar{y}$ is a s.c. element of order 5 in $\bar{C}$ and $C(t) \cap C(y)=\langle t y\rangle$ in this case as well. In all cases $t^{y_{1}} \in C(t) \cap C(y)=$ $\langle t y\rangle$ so $t^{y_{1}}=t$. Hence $y_{1} \in C(t y)=\langle t y\rangle$ and $y_{1} \in\langle y\rangle$, a contradiction proving the claim. A recent result of $M$. Aschbacher [1] now gives the possibilities for $G$. We see that there is no additional group satisfying our conditions. This completes the proof of the theorem.

\section{REFERENCES}

1. M. Aschbacher, Thin finite simple groups, Bull. Amer. Math. Soc. 82 (1976), 484.

2. W. Feit and J. G. Thompson, Finite groups which contain a self-centralizing subgroup of order 3, Nagoya Math. J. 21 (1962), 185-197.

3. D. Gorenstein, Centralizers of involution of finite simple groups, Finite Simple Groups, Academic Press, London, 1971.

4. __ Finite groups, Harper and Row, New York, 1968.

5. D. Gorenstein and K. Harada, Finite groups whose 2-subgroups are generated by at most 4 elements, Mem. Amer. Math. Soc. No. 147, 1974.

6. G. Higman, Odd characterizations of finite simple groups, (Lecture notes, University of Michigan, 1968).

7. Z. Janko, A new finite simple group with Abelian Sylow 2-subgroups and its characterization, J. Algebra 3 (1966), 147-186.

8. $\ldots, A$ class of simple groups of characteristic 2, Finite Groups' 72, North-Holland, New York, 1973.

9. Z. Janko and J. G. Thompson, On a class of finite simple groups of Ree, J. Algebra 4 (1966), 274-292.

10. L. Schiefelbusch, Sylow 2-subgroups of simple groups, J. Algebra 31 (1974), 131-153.

11. I. Schur, Untersuchungen über die Darstellung der endlichen Gruppen durch gebrochene lineare Substitutionen, J. für die r. und a Math. 132 (1907), 85-137.

12. M. Suzuki, On finite groups containing an element of order four which commutes only with its powers, Illinois J. Math. 3 (1959), 255-271.

Department of Mathematics, Bowling Green State University, Bowling Green, Oho 43403

Department of Mathematics, Michigan State University, East Lansing, Michigan 48824 